\title{
Exotic States in Long-Range Spin Glasses
}

\author{
A. Gandolfi, ${ }^{1,2}$, C.M. Newman ${ }^{1,3}$, and D.L. Stein ${ }^{4,5}$ \\ ${ }^{1}$ Research supported in part by NSF Grants DMS-9196086 and 9209053 \\ 2 Dept. of Statistics, Univ. of California, Berkeley, CA 94720, USA \\ 3 Courant Institute of Mathematical Sciences, New York University, New York, NY 10012, USA \\ ${ }^{4}$ Research supported in part by U.S. Department of Energy Grant No. DE-FG03-93ER25155 \\ 5 Department of Physics, University of Arizona, Tucson, AZ 85721, USA
}

Received November 17, 1992

\begin{abstract}
We consider Ising spin glasses on $\mathbf{Z}^{d}$ with couplings $J_{x y}=c_{y-x} Z_{x y}$, where the $c_{y}$ 's are nonrandom real coefficients and the $Z_{x y}$ 's are independent, identically distributed random variables with $E\left[Z_{x y}\right]=0$ and $E\left[Z_{x y}^{2}\right]=1$. We prove that if $\sum_{y}\left|c_{y}\right|=\infty$ while $\sum_{y}\left|c_{y}\right|^{2}<\infty$, then (with probability one) there are uncountably many (infinite volume) ground states $\tilde{\sigma}$, each of which has the following property: for any temperature $T<\infty$, there is a Gibbs state supported entirely on (infinite volume) spin configurations which differ from $\tilde{\sigma}$ only at finitely many sites. This and related results are examples of the bizarre effects that can occur in disordered systems with coupling-dependent boundary conditions.
\end{abstract}

\section{Introduction}

The majority of theoretical work on realistic (i.e., non-infinite-range) spin glasses has focused on the nearest-neighbor Edwards-Anderson (EA) model [1,2], primarily due to the relatively simple form of its Hamiltonian. A smaller body of work has studied models with random long-range interactions which are square summable; the usual case is that of power-law decay. These models are of interest for several reasons: the one-dimensional case is partially tractable and is believed to display a phase transition for a certain range of values of the power-law exponent [3]; there is a significant body of rigorously provable results (see [4] and references therein); and in three dimensions, models with a $1 / r^{3}$ falloff approximate more closely than nearest-neighbor models the RKKY interactions within an important class of laboratory spin glasses (specifically, dilute magnetic alloys) [2].

It is useful to distinguish among four different classes of spin glass models (we confine ourselves to Ising systems): 1) the infinite-range Sherrington-Kirkpatrick (SK) model [5]; 2) the nearest-neighbor EA model (or other short-range models); 3) models with long-range random interactions of the kind discussed above; and 4) randomly site-diluted models with deterministic interactions of non-constant sign, such as RKKY. Of the above, only the first is fairly well-understood (but mostly on a non-rigorous basis); whether its properties apply to any of the other three is 
a subject of much debate. Of the latter three, (2) appears to be the simplest; importantly, it is generally believed that its basic thermodynamic properties (presence or absence of a phase transition, multiplicity of ground states) resemble those of (3) and (4), as well as those of laboratory spin glasses (see for example, Ref. 6). However, the extent to which all of these models resemble or differ from one another remains unclear.

In this paper we focus exclusively on models of class (3). We find, essentially through judicious choices of coupling-dependent boundary conditions, that an uncountable number of Gibbs states can be generated at any temperature. These Gibbs states and their associated ground states will have unusual properties to be discussed in the next section. Moreover, we find an uncountable number of even more exotic ground state spin configurations, with the following property - the flip of any finite subset of spins costs an infinite amount of energy. This last property cannot extend to short-range models; neither can the property of having many Gibbs states at high temperature. Nevertheless, these findings lead to important questions which are relevant to short-range spin glasses.

To motivate the discussion in the next section, we consider here a onedimensional model with the following formal Hamiltonian:

$$
\mathscr{H}=-\frac{1}{4} \sum_{\substack{x, y \in \mathbf{Z} \\ x \neq y}} \frac{Z_{x y}}{|x-y|^{\alpha}} \sigma_{x} \sigma_{y},
$$

where the $Z_{x y}\left(=Z_{y x}\right)$ are independent, identically distributed random variables with mean zero and variance one, $1 / 2<\alpha \leqq 1$, and the $\sigma_{x}$ are \pm 1 valued Ising spins. If we confine our attention to a finite system within a volume $\Lambda$ with a boundary condition $\sigma^{b}$ outside this volume, the Hamiltonian becomes

$$
\mathscr{H}=-\frac{1}{4} \sum_{\substack{x, y \in \Lambda \\ x \neq y}} \frac{Z_{x y}}{|x-y|^{\alpha}} \sigma_{x} \sigma_{y}-\frac{1}{2} \sum_{\substack{x \in \Lambda \\ y \notin \Lambda}} \frac{Z_{x y}}{|x-y|^{\alpha}} \sigma_{x} \sigma_{y}^{b} .
$$

It is known [7] that for any $\sigma^{b}$ which does not depend on the $Z_{x y}$ 's the free energy per spin exists and is a constant independent of $\left\{Z_{x y}\right\}$ and of the boundary condition $\sigma^{b}$. It has also been proved [8] that for $\alpha>1$, there is "weak uniqueness" (see also Ref. 9) of the Gibbs state for any nonzero temperature; i.e., for any $\sigma^{b}$ as above, there is (with probability one) a single infinite-volume limit Gibbs state for any sequence of volumes tending to $\mathbf{Z}$, and if $\sigma^{b \prime}$ is another coupling-independent boundary condition, then the same infinite-volume Gibbs state results. Furthermore, this state is not a mixture of other Gibbs states. For the range $1 / 2<\alpha \leqq 1$, weak uniqueness of Gibbs states is known to be valid for sufficiently high temperature [10]. Although non-rigorous arguments indicate that this model has a phase transition [3], there are no rigorous results which extend to low temperatures; i.e., there is as yet no proof that weak uniqueness breaks down at low temperatures.

We now give a simple argument to indicate that unusual things can happen when coupling-dependent boundary conditions are allowed. For illustrative purposes, consider a one-dimensional system with Hamiltonian (1.2) (but restricted to the right half-line), and with $\Lambda=[1, L]$. Focus on a spin at site $x$, with $1 \leqq|x| \leqq L$. 
The energy contribution of this spin can be written as $-h_{x} \sigma_{x}$, where

$$
\begin{aligned}
h_{x} & =\frac{1}{2} \sum_{y(\neq x) \leqq L} \frac{Z_{x y}}{|x-y|^{\alpha}} \sigma_{y}+\frac{1}{2} \sum_{y>L} \frac{Z_{x y}}{|x-y|^{\alpha}} \sigma_{y}^{b} \\
& =\frac{1}{2} \sum_{y(\neq x) \leqq L} \frac{Z_{x y}}{|x-y|^{\alpha}} \sigma_{y}+\frac{1}{2} \sum_{\substack{y=x+n L \\
n=1,2, \ldots, \infty}} \frac{Z_{x y}}{|x-y|^{\alpha}} \sigma_{y}^{b} \\
& +\frac{1}{2} \sum_{\substack{L<y \neq x+n L \\
n=1,2, \ldots, \infty}} \frac{Z_{x y}}{|x-y|^{\alpha}} \sigma_{y}^{b} .
\end{aligned}
$$

Consider the three terms which combine to give $h_{x}$. The first is of course finite. If the $\sigma_{y}^{b}$ 's of the third term are chosen independently of the $Z_{x y}$ 's appearing in that term, then it follows that (with probability one) the sum converges and hence the third term is also finite. (The convergence of the sum for $\alpha>1 / 2$ will be discussed in Sect. 3.) But now consider what happens when the remaining boundary spins which appear in the second term are chosen in a certain coupling-dependent manner. In particular, suppose we make the boundary-dependent choice $\sigma_{y}^{b}=\operatorname{sgn}\left(Z_{x y}\right)$ for each $\sigma_{y}^{b}$ in the second term; then the resulting sum will diverge for $\alpha \leqq 1$ and it would cost infinite energy to flip $\sigma_{x}$ from +1 to -1 . Similarly, by choosing $\sigma_{y}^{b}=-\operatorname{sgn}\left(Z_{x y}\right)$, it would cost infinite energy to flip $\sigma_{x}$ from -1 to +1 .

We have not presented a detailed version of this argument because our purpose in this introduction is only to motivate the assertion that we can always choose boundary conditions to force any particular spin $\sigma_{x}$ with $x \in \Lambda$ to point either up or down in the ground state, such that a flip would cost infinite energy. Furthermore, it is clear that we can repeat the same procedure for all of the spins in $\Lambda$. The only potential problem is the third term, but that will remain finite because of the independence of the $Z_{x y}$ 's. That is, a $\sigma_{y}^{b}$ which depends on a particular $Z_{x y}$ is still independent of the other couplings.

A similar line of reasoning implies that boundary conditions can be chosen to make a spin configuration as "stiff" as desired; for each $x$ in $\Lambda$, we can choose the boundary conditions to create a large (but finite) field $h_{x}$ of any desired sign and above any desired magnitude by cutting off the choice $\sigma_{y}^{b}=\operatorname{sgn}\left(Z_{x y}\right)$ after a sufficiently large number of terms. That is, one can arrange the boundary spin configuration to force a (finite volume) ground state with the property that overturning any spin will cost a finite but large (of any magnitude desired) energy.

These rather crude arguments provide some idea of the kinds of situations which can arise when coupling-dependent boundary conditions are employed. They do not comprise a satisfactory picture because they don't provide a procedure for generating infinite-volume Gibbs states (or ground state spin configurations) with these exotic properties. Do such states actually exist? We will prove in the following sections that not only is the answer yes, but an uncountable number exist, and this is the case at all temperatures.

An immediate question then is what effect, if any, such exotic states have on "observable" properties. Since states which do not "arise" except from couplingdependent boundary conditions may be regarded as not being physically observable [9], one interpretation of this question [11] is whether these exotic states do somehow arise at low temperature when using, say, free boundary conditions. Another question is, does any of this shed light on nearest-neighbor models? Because we have only speculations to offer at this point, we defer a treatment of 
these issues to the future. We would however like to make one point, which is developed in much greater detail in Ref. 12 in the context of short- and infiniterange models. That is that there are two ways in which multiple (exotic) states could arise from, say, free boundary conditions. The first (but, as argued in Ref. 12, rather unlikely way) is that there is a single, well-defined infinite volume limit, whose decomposition into extremal Gibbs states could include our exotic states. The second way is that the exotic states could be included among many different limits along different subsequences of volumes. Even though singling out a particular exotic state would require a coupling-dependent subsequence of volumes, the multiple states would be observable in their contibution to the sensitive dependence on volumes of free boundary condition states.

The rest of the paper is organized as follows: Sect. 2 contains a statement of four theorems, along with some discussion, that assert the existence of exotic Gibbs states and delineate their properties. Sections 3, 4, and 5 contain the proofs of Theorems 2.1, 2.2, and 2.4, respectively. The proof of Theorem 2.3 is straightforward and is discussed in Sect. 2.

\section{Main Results}

We consider a formal Hamiltonian, $\mathscr{H}$, for Ising spin configurations on $\mathbf{Z}^{d}$ :

$$
\mathscr{H}=-\frac{1}{2} \sum_{\{x, y\}} J_{x y} \sigma_{x} \sigma_{y},
$$

where the sum is over all pairs of sites in $\mathbf{Z}^{d}$, the spins $\sigma_{x}$ take values +1 or -1 , and each $J_{x y}$ is a real number (with $J_{x y}=J_{y x}$ and $J_{x x}=0$ ). To define infinite volume ground states (or, later on, Gibbs states) when, for fixed $x, \sum_{y}\left|J_{x y}\right|$ diverges, we must specify an order of summation to take advantage of possible conditional convergence of various series. Thus we will fix, once and for all, (for any choice of $J_{x y}$ 's) an increasing sequence $\Lambda_{n}$ of finite subsets of $\mathbf{Z}^{d}$ tending to all of $\mathbf{Z}^{d}$ as $n \rightarrow \infty$. For simplicity, we choose

$$
\Lambda_{n}=\left\{x \in \mathbf{Z}^{d}:|x| \leqq n\right\}=\{-n,-n+1, \ldots, n\}^{d}
$$

where $|x|=\sup _{1 \leq i \leq d}\left|x_{i}\right|$.

An infinite-volume ground state is a configuration of spins on $\mathbf{Z}^{d}$ with the property that the flip of any finite subset of spins raises the energy. More precisely, an infinite-volume ground state will be defined as any spin configuration $\sigma$ (mapping each $x$ in $\mathbf{Z}^{d}$ to $\sigma_{x}=+1$ or -1 ) such that for every finite subset $A$ of $\mathbf{Z}^{d}$,

$$
\Delta \mathscr{H}_{A}(\sigma) \equiv \lim _{n \rightarrow \infty}\left(\sum_{x \in A} \sum_{y \in \Lambda_{n} \backslash A} J_{x y} \sigma_{x} \sigma_{y}\right) \geqq 0 .
$$

This definition requires the above limit to exist for each finite $A$, but we allow the limit to be $+\infty$. (When $A$ is the empty set, $\Delta \mathscr{H}_{A}$ is defined to be zero.) The limit represents the energy cost of flipping the spins in $A$ while leaving all others fixed. The relation of this definition to that of finite and infinite volume Gibbs states will be discussed below, where we will also consider the "stiffness" of ground states based on the magnitude of the $\Delta \mathscr{H}_{A}$ 's. But first, we focus on the stiffest possible ground state, which we call a rigid ground state. This is one for which 
$\Delta \mathscr{H}_{A}=+\infty$ for every nonempty finite $A$. Equivalently, it suffices to require that for each $x$ in $\mathbf{Z}^{d}$,

$$
\Delta \mathscr{H}_{x}(\sigma) \equiv \lim _{n \rightarrow \infty} \sum_{y \in \Lambda_{n}} J_{x y} \sigma_{x} \sigma_{y}=+\infty .
$$

Clearly, $\sum_{y}\left|J_{x y}\right|$ must diverge for each $x$ in order that rigid ground states might exist. When the couplings are all positive, then the identically +1 and identically -1 configurations are both rigid. Sometimes these are the only rigid ground states. Consider, for example, the case when $d=1$ and $J_{x y}=|y-x|^{-\alpha}$ with $\alpha \leqq 1$. (This example is rather artificial in that the free energy per site is infinite in the thermodynamic limit even with free boundary conditions, but we use it simply as an illustration.) For any $\alpha>0$,

$$
\sum_{\substack{y \in A_{n} \\ y \neq x_{1}}} \frac{1}{\left|y-x_{1}\right|^{\alpha}} \sigma_{y}-\sum_{\substack{y \in A_{n} \\ y \neq x_{2}}} \frac{1}{\left|y-x_{2}\right|^{\alpha}} \sigma_{y}=O(1),
$$

which implies that in a rigid ground state, $\sigma_{x_{1}}=\sigma_{x_{2}}$ for every $x_{1}, x_{2}$. If one multiplies this $d=1$ coupling by the deterministic sign $\varepsilon_{x y}=(-1)^{|x-y|}$, then one obtains a model equivalent to the ferromagnetic one (under the gauge transformation of flipping all $\sigma_{x}$ 's with $x$ odd) so there are still exactly two rigid ground states. However, if one instead uses the sign $\tilde{\varepsilon}_{x y}=(-1)^{|x-y|+1}$, then the model is equivalent to a fully antiferromagnetic one which, by again using Eq. (2.5), can easily be seen to have no rigid ground states.

When the signs of the couplings are chosen at random, the existence and number of rigid ground states is a priori unclear. The next theorem shows that if the couplings of the above $d=1$ example are multiplied by random signs and if $\frac{1}{2}<\alpha \leqq 1$, then there are uncountably many rigid ground states (for almost every choice of the random signs).

Theorem 2.1. For pairs $\{x, y\}$ of distinct sites in $\mathbf{Z}^{d}$, let $Z_{x y}\left(=Z_{y x}\right)$ be independent identically distributed random variables whose common distribution has zero mean and variance equal to one. Let $c_{y}$ be a (nonrandom) sequence of real numbers indexed by $y$ in $\mathbf{Z}^{d}$ (with $\left.c_{0}=0\right)$ such that

$$
\sum_{y \in \mathbf{Z}^{d}}\left|c_{y}\right|=\infty \text { and } \sum_{y \in \mathbf{Z}^{d}}\left|c_{y}\right|^{2}<\infty,
$$

and let $J_{x y}=c_{y-x} Z_{x y}$ in the Hamiltonian (2.1). Let $S$ be any subset of $\mathbf{Z}^{d}$ with the property that

$$
\text { for each } x \text { in } \mathbf{Z}^{d}, \quad \sum_{y \in S}\left|c_{y-x}\right|<\infty .
$$

(An infinite such $S$ always exists.) Then, with probability one, for every choice of a spin configuration $\hat{\sigma}$ on $S$ there is a rigid ground state $\sigma^{*}$ which agrees with $\hat{\sigma}$ on all of $S$; i.e.,

$$
\sigma_{w}^{*}=\hat{\sigma}_{w} \text { for each } w \text { in } S .
$$

The proof of Theorem 2.1 will be given below in Sect. 3 of the paper. It is based on decomposing $\mathbf{Z}^{d}$ into a disjoint union of directed trees, one starting from each $w$ in $S$. Within each tree the spin values are defined inductively, starting from the prescribed $\hat{\sigma}_{w}$ on the root $w$ of the tree, so that the spin $\sigma_{y}^{*}$ equals $\operatorname{sgn}\left(J_{x y}\right) \cdot \sigma_{x}^{*}$, where $x$ is the immediate predecessor of $y$ on the tree. Rigid ground states are 
obtained by constructing suitable trees with infinitely many branches coming out of each $x$. The terms in the Hamiltonian corresponding to these different branches (from a single $x$ ) add coherently, while other terms from within a given tree or between trees add incoherently. A modification of this construction (which we give in Sect. 4), in which a large but finite number of branches come from each $x$ leads to ground states in which $\Delta \mathscr{H}_{A}$ may be made arbitrarily large (but finite) for each nonempty $A$.

Let us then define a pliable ground state as one in which $\Delta \mathscr{H}_{A}<\infty$ for each finite $A$. (We denote henceforth by $\mathscr{A}$ the set of all finite subsets $A$ of $\mathbf{Z}^{d}$.) Further, given any sequence $\theta=\left(\theta_{A}: A \in \mathscr{A}\right)$ of non-negative numbers indexed by $A$, we will call a ground state $\sigma, \theta$-stiff if

$$
\triangle \mathscr{H}_{A}(\sigma) \geqq \theta_{A} \quad \text { for every } A \in \mathscr{A} .
$$

Theorem 2.2. Let $\theta=\left(\theta_{A}: A \in \mathscr{A}\right)$ be any non-negative sequence indexed by $A \in \mathscr{A}$ (with $\theta$ of the empty set equal to zero) and assume the same hypotheses as in Theorem 2.1. Then, with probability one, for every choice of a spin configuration $\hat{\sigma}$ on $S$, there is a pliable, $\theta$-stiff ground state $\tilde{\sigma}$ which agrees with $\hat{\sigma}$ on all of $S$.

In order to explore the consequences of Theorem 2.2 for the nature of Gibbs states, we need to define finite and infinite volume Gibbs states at inverse temperature $\beta<\infty$. First we define a spin configuration $\sigma$ (on all of $\mathbf{Z}^{d}$ ) to be allowed (as a boundary condition for every finite region $\Lambda \in \mathbf{Z}^{d}$ ) if for every $x$ in $\mathbf{Z}^{d}$,

$$
h_{x}(\sigma) \equiv \frac{1}{2} \lim _{n \rightarrow \infty} \sum_{y \in \Lambda_{n}} J_{x y} \sigma_{y} \quad \text { exists and is finite. }
$$

(Note that a pliable ground state is always allowed.) Then, we can define for any finite $\Lambda \in \mathbf{Z}^{d}$, the interaction energy between a spin configuration $\sigma^{\Lambda}$ on $\Lambda$ and the allowed boundary condition $\sigma$ as

$$
\begin{aligned}
\mathscr{H}_{I}^{\Lambda}\left(\sigma^{\Lambda} ; \sigma\right) & =-\frac{1}{2} \lim _{n \rightarrow \infty} \sum_{x \in \Lambda} \sum_{y \in \Lambda_{n} \backslash \Lambda} J_{x y} \sigma_{x}^{\Lambda} \sigma_{y} \\
& =+\frac{1}{2} \sum_{x, y \in \Lambda} J_{x y} \sigma_{x}^{\Lambda} \sigma_{y}-\sum_{x \in \Lambda} h_{x}(\sigma) \sigma_{x}^{\Lambda},
\end{aligned}
$$

and the "total" energy of $\sigma^{\Lambda}$, for the boundary condition $\sigma$ as

$$
\mathscr{H}^{\Lambda}\left(\sigma^{\Lambda} ; \sigma\right)=-\frac{1}{4} \sum_{x, y \in \Lambda} J_{x y} \sigma_{x}^{\Lambda} \sigma_{y}^{\Lambda}+\mathscr{H}_{I}^{\Lambda}\left(\sigma^{\Lambda} ; \sigma\right) .
$$

A finite volume Gibbs state in the region $\Lambda$ at inverse temperature $\beta \in[0, \infty)$ with (allowed) boundary condition $\sigma$ is the probability measure $\rho_{\beta, \sigma}^{A}$ on configurtions $\sigma^{\Lambda}$ with

$$
\rho_{\beta, \sigma}^{\Lambda}\left(\left\{\sigma^{\Lambda}\right\}\right)=\left(Z_{\beta, \sigma}^{\Lambda}\right)^{-1} \exp \left(-\beta \mathscr{H}^{\Lambda}\left(\sigma^{\Lambda} ; \sigma\right)\right),
$$

where

$$
Z_{\beta, \sigma}^{\Lambda}=\sum_{\sigma^{\Lambda}} \exp \left(-\beta \mathscr{H}^{\Lambda}\left(\sigma^{\Lambda} ; \sigma\right)\right) .
$$

Note that this, of course, depends only on the $\sigma_{y}$ 's for $y \notin \Lambda$. 
A probability measure $\rho$ on configurations $\sigma^{\prime}$ (on all of $\mathbf{Z}^{d}$ ) will be called an infinite volume Gibbs state at inverse temperature $\beta \in[0, \infty)$ if

$$
\rho(\{\text { allowed } \sigma \text { 's }\})=1
$$

and for every finite $\Lambda \subset \mathbf{Z}^{d}$, the conditional probability that $\sigma_{x}^{\prime}=\sigma_{x}^{A}$ for each $x \in \Lambda$, given (the $\sigma$-field generated by) $\left\{\sigma_{y}: y \notin \Lambda\right\}$ is

$$
\rho\left(\sigma^{\Lambda} \mid\left\{\sigma_{y}: y \notin \Lambda\right\}\right)=\rho_{\beta, \sigma}^{\Lambda}\left(\left\{\sigma^{\Lambda}\right\}\right)
$$

for $\rho$-almost all $\sigma$ 's.

In general, if one takes a boundary condition $\tilde{\sigma}$ to be a pliable ground state, then the limit (or limits), as $\Lambda \rightarrow \mathbf{Z}^{d}$, of $\rho_{\beta, \tilde{\sigma}}^{A}$ will be supported on infinite volume spin configurations $\sigma$ which differ from $\tilde{\sigma}$ at infinitely many sites (as in, e.g., the standard nearest neighbor Ising ferromagnet). However, if $\tilde{\sigma}$ is sufficiently stiff, in particular if

$$
\sum_{A \in \mathscr{A}} e^{-\beta \Delta H_{A}(\tilde{\sigma})}<\infty
$$

the situation will be quite different: the limit will be the infinite-volume Gibbs state described in the next proposition, which is supported entirely on $\sigma$ 's which differ from $\tilde{\sigma}$ at only finitely many sites. The relevance of this fact is that, by Theorem 2.2, we can construct uncountably many $\tilde{\sigma}$ 's such that (2.17) is valid for all $\beta>0$. To see this, simply order the elements of $\mathscr{A}$ as $A_{1}, A_{2}, \ldots$ and choose $\theta_{k}=\theta_{A_{k}}$ so that $\theta_{k} / \ln (k) \rightarrow \infty$ as $k \rightarrow \infty$.

We leave the proof of the next theorem to the reader; it is basically just a matter of disentangling definitions.

Theorem 2.3. Suppose $\tilde{\sigma}$ is a pliable ground state such that (2.17) is valid for some $\beta \in(0, \infty)$. Define a probability measure $\rho_{\beta}^{\tilde{\sigma}}$ on infinite volume spin configurations by

$$
\rho_{\beta}^{\tilde{\sigma}}\left(\left\{\tilde{\sigma}^{A}\right\}\right)=e^{-\beta \Delta H_{A}(\tilde{\sigma})} / \sum_{A \in \mathscr{A}} e^{-\beta \Delta H_{A}(\tilde{\sigma})}, \quad A \in \mathscr{A},
$$

where $\tilde{\sigma}^{A}$ denotes the configuration obtained from $\tilde{\sigma}$ by flipping all the sites in $A$. Then $\rho_{\beta}^{\tilde{\sigma}}$ is an infinite-volume Gibbs distribution at inverse temperature $\beta$.

The final topic we consider in this section is the use of a spin configuration $\sigma^{\prime}$ on $\mathbf{Z}^{d}$ as a boundary condition when, for each $x$, the limit in (2.10) exists but is infinite (either $+\infty$ or $-\infty$ ). We will say (for reasons that will be clarified below) that such a spin configuration $\sigma^{\prime}$ is forcing. One type of example is when $\sigma^{\prime}$ is a rigid ground state, in which case the sign of infinity for $h_{x}\left(\sigma^{\prime}\right)$ is just $\sigma_{x}^{\prime}$ itself, for each $x$. Such a configuration is not an allowed boundary condition (according to our definition) but nevertheless one may construct a finite volume measure on configurations $\sigma^{A}$ by first replacing $\mathbf{Z}^{d}$ by $\Lambda_{n}$ (i.e., by taking $\sigma^{\prime}$ as boundary condition in $\Lambda_{n} \backslash \Lambda$ and free boundary conditions on $\mathbf{Z}^{d} \backslash \Lambda_{n}$ ), considering the resulting Gibbs state $\rho_{\beta, \sigma^{\prime}, \Lambda_{n}}^{A}$ and then taking the limit as $n \rightarrow \infty$.

If $\sigma^{\prime}$ is a rigid ground state, then it is clear that for any $\beta>0$, the resulting measure, which we denote (as in the allowed case) $\rho_{\beta, \sigma^{\prime}}^{A}$ is simply a Dirac measure on the single configuration $\sigma^{\Lambda}$ with $\sigma_{x}^{A}=\sigma^{\prime}$ for each $x$ in $\Lambda$. For a general forcing $\sigma^{\prime}$, the measure $\rho_{\beta, \sigma^{\prime}}^{\Lambda}$ is supported on the single configuration with $\sigma_{x}^{\Lambda}=\operatorname{sgn}\left(h_{x}\left(\sigma^{\prime}\right)\right)$ for each $x$ in $\Lambda$. The limit as $\Lambda \rightarrow \mathbf{Z}^{d}$ of $\rho_{\beta, \sigma^{\prime}}^{A}$ is of course simply the Dirac measure on $\sigma$ with $\sigma_{x}=\operatorname{sgn}\left(h_{x}\left(\sigma^{\prime}\right)\right)$ for each $x$ in $\mathbf{Z}^{d}$; naturally, we will say that $\sigma^{\prime}$ forces $\sigma$. 
Since a rigid ground state $\sigma^{*}$ forces itself, it is possible to regard the Dirac measure on $\sigma^{*}$ as a kind of (degenerate version of) an infinite volume Gibbs state (for any inverse temperature $\beta>0$ ). This is certainly not so for a Dirac measure on a configuration $\sigma$ which is not a rigid ground state. Thus if for some $\sigma$ which is not a rigid ground state, there exists a $\sigma^{\prime}$ which forces $\sigma$, then we see that coupling dependent boundary conditions (since the construction of such a $\sigma^{\prime}$ will depend on the $J_{x y}$ 's) can yield infinite volume limits which are degenerate and not Gibbs states (even in the degenerate sense). This leads us to ask whether there are any (or many) configurations $\sigma$, other than rigid ground states, which can be forced by some $\sigma^{\prime}$. The next theorem gives the rather striking answer. Its proof (which is a bit simpler than those of the other theorems) is given in Sect. 5.

Theorem 2.4. Let $J_{x y}$ be given as in Theorem 2.1. Then with probability one, for every spin configuration $\sigma$ on $\mathbf{Z}^{d}$, there is some configuration $\sigma^{\prime}$ on $\mathbf{Z}^{d}$ which forces $\sigma$.

Remark. In fact, the above result is easily strengthened to show that (with probability one) there are, for every $\sigma$, uncountably many configurations $\sigma^{\prime}$ which force $\sigma$. To see that, choose any infinite $S$ satisfying Eq. (2.7). Then it is easy to see that (with probability one) for every $x$ in $\mathbf{Z}^{d}, \sum_{y \in S}\left|J_{x y}\right|<\infty$. It follows that each forcing pair $\left(\sigma^{\prime}, \sigma\right)$ yields an uncountable equivalence class of forcing pairs in which $\sigma^{\prime}$ is changed arbitrarily on $S$, while $\sigma$ remains the same.

\section{Proof of Theorem 2.1}

The general strategy will be to choose (in a manner described below) for each $x \in \mathbf{Z}^{d}$ an infinite subset $U_{x}$ of $\mathbf{Z}^{d}$ such that the $U_{x}$ 's are pairwise disjoint with

$$
\sum_{y \in U_{x}}\left|c_{y-x}\right|=\infty, \text { for all } x \in \mathbf{Z}^{d} .
$$

Consider the directed graph with vertex set $\mathbf{Z}^{d}$ and a directed edge from $x$ to $y$ whenever $y \in U_{x}$. This will be a union of disjoint trees. We will require each tree to have a root (a site not in any $U_{x}$ ) and the set of roots to coincide exactly with the set $S$ given in the theorem. For $w \in S$, let $T_{w}$ denote the tree with root $w$. Once we have chosen the spin value of each root according to Eq. (2.8), all other spins will be uniquely determined as

$$
\sigma_{y}^{*}=\sigma_{w}^{*} \eta_{y} \text { for all } y \in T_{w},
$$

where $\eta_{y}$ is defined inductively, by setting $\eta_{w}=+1$ for each $w \in S$ and requiring:

$$
\text { for each } x \in \mathbf{Z}^{d}, \quad \eta_{y}=\operatorname{sgn}\left(J_{x y} \eta_{x}\right) \quad \text { for every } y \in U_{x} \text {. }
$$

(Here $\operatorname{sgn}(0)$ may be taken to be +1 .)

In addition to Eq. (3.1), a crucial property of our partition of $\mathbf{Z}^{d}$ into the disjoint tree set $\left\{T_{w}: w \in S\right\}$ will be that

$$
\sum_{w \in S}\left[\sum_{y \in T_{w}}\left|c_{y-x}\right|^{2}\right]^{1 / 2}<\infty \quad \text { for all } x \in \mathbf{Z}^{d} .
$$

The construction of the $U_{x}$ 's and the decomposition of $\mathbf{Z}^{d}$ into the trees, $T_{w}$, satisfying Eqs. (3.1) and (3.4) will be given below. We proceed to complete the proof of Theorem (2.1) given such a decomposition. 
Let us first show that for any single choice of $\hat{\sigma}$, the resulting $\sigma^{*}$ (given by Eqs. (3.2)-(3.3)) is a rigid ground state with probability one. To do this we must show that for each $x \in \mathbf{Z}^{d}$,

$$
\Delta H_{x}\left(\sigma^{*}\right) \equiv \lim _{n \rightarrow \infty} \sum_{y \in \Lambda_{n}} J_{x y} \sigma_{x}^{*} \sigma_{y}^{*}=\infty
$$

with probability one. We express $\Delta H_{x}\left(\sigma^{*}\right)$ as the sum of two terms $V$ and $V^{\prime}$. In $V^{\prime}$ we restrict $y$ to be either in $U_{x}$ or (if $x \notin S$ ) to include the (unique) $y_{0}$ with $x \in U_{y_{0}}$. In $V$ we take all other $y$ 's (both those in the same tree as $x$ and those in different trees); $V=\lim _{n \rightarrow \infty} V^{(n)}$, where in $V^{(n)}, y$ is further restriced to be in $\Lambda_{n}$.

First we note that for the $y^{\prime}$ s of $V^{\prime}$,

$$
J_{x y} \sigma_{x}^{*} \sigma_{y}^{*}=J_{x y} \eta_{x} \eta_{y}=J_{x y} \operatorname{sgn}\left(J_{x y}\right)=\left|J_{x y}\right|,
$$

so that (with probability one) $V^{\prime}=\infty$ because

$$
\sum_{y \in U_{x}}\left|J_{x y}\right|=\sum_{y \in U_{x}}\left|c_{y-x}\right|\left|Z_{x y}\right|=\infty .
$$

The divergence of Eq. (3.7) follows from Eq. (3.1) because the $\left|Z_{x y}\right|$ 's are i.i.d. (and not identically zero) non-negative random variables. (This follows, for example, from the Kolmogorov three-series theorem; see, e.g., Chung [13].) Next we note that for the $y^{\prime}$ s of $V, J_{x y} \sigma_{x}^{*} \sigma_{y}^{*}=c_{x-y} Z_{x y} \sigma_{x}^{*} \sigma_{y}^{*}$, with the random variables $\left\{\sigma_{x}^{*} \sigma_{y}^{*}\right\}$ independent of the random variables $\left\{Z_{x y}\right\}$ because the signs $\sigma_{x}^{*} \sigma_{y}^{*}$ are defined only in terms of other $Z_{x^{\prime} y^{\prime}}$ 's. Since the $Z_{x y}$ 's are independent, mean zero and since $E\left(V^{(n) 2}\right)$ is uniformly bounded by $\sum_{y}\left|c_{x-y}\right|^{2}<\infty$, the existence and finiteness (with probability one) of the limit defining $V$ follows by a martingale convergence theorem (see e.g., Hall and Heyde [14], Corollary 2.2). This proves that for any single choice of $\hat{\sigma}$, Eq. (3.5) is valid with probability one.

To complete the proof, we must show (for each $x \in \mathbf{Z}^{d}$ ) that, with probability one, Eq. (3.5) is (simultaneously) valid for all choices of $\hat{\sigma}$. By Eq. (3.6), $V^{\prime}$ does not depend on $\hat{\sigma}$ and is still $+\infty$ as before, but $V=V(\hat{\sigma})$ must be shown (with probability one) to be finite for all choices of $\hat{\sigma}$. To do this we write $V$ as a sum, where each term only includes $y$ 's from a single tree, $T_{w}$. Denoting by $w(x)$ the root of the tree containing $x$, we have

$$
V=\lim _{n \rightarrow \infty} \sum_{w \in S} \hat{\sigma}_{w(x)} \hat{\sigma}_{w} V_{w}^{(n)} .
$$

For $w=w(x)$

$$
V_{w(x)}^{(n)}=\sum_{y \in \tilde{T}_{w(x)} \cap A_{n}} c_{x-y} Z_{x y} \eta_{x} \eta_{y},
$$

where $\tilde{T}_{w(x)}$ is $T_{w(x)}$, except for the $y$ 's included in $V^{\prime}$. For $w \neq w(x)$,

$$
V_{w}^{(n)}=\sum_{y \in T_{w(x)} \cap \Lambda_{n}} c_{x-y} Z_{x y} \eta_{x} \eta_{y} .
$$

To prove convergence of (3.8) to a finite limit for all choices of $\hat{\sigma}$, it suffices, by the dominated convergence theorem, to show that (with probability one), each $V_{w}^{(n)}$ has a limit as $n \rightarrow \infty$ and that

$$
\sum_{w \in S} \sup _{n \geqq 0}\left|V_{w}^{(n)}\right|<\infty .
$$


The limit for each $V_{w}^{(n)}$ follows by the same martingale convergence argument as given above for the limit defining $V$ (for a single $\hat{\sigma}$ ). To obtain Eq. (3.11), it suffices if

$$
\sum_{w \in S} E\left(\sup _{n \geqq 0}\left|V_{w}^{(n)}\right|\right)<\infty
$$

But then,

$$
\begin{aligned}
E\left(\sup _{n \geqq 0}\left|V_{w}^{(n)}\right|\right) & =\lim _{n \rightarrow \infty} E\left(\sup _{n \geqq k \geqq 0}\left|V_{w}^{(k)}\right|\right) \\
& \leqq \lim _{n \rightarrow \infty}\left[E\left(\sup _{n \geqq k \geqq 0}\left|V_{w}^{(k)}\right|^{2}\right)\right]^{1 / 2} \\
& \leqq \lim _{n \rightarrow \infty} \sqrt{2}\left[E\left(V_{w}^{(n) 2}\right)\right]^{1 / 2} \\
& \leqq \sqrt{2}\left[\sum_{y \in T_{w}}\left|c_{y-x}\right|^{2}\right]^{1 / 2}
\end{aligned}
$$

where the first inequality is Cauchy-Schwarz and the second is Doob's inequality for martingales (see Hall and Heyde [14], Theorem 2.2). Combining Eq. (3.13) with (3.4) yields (3.12) as desired. The proof of Theorem 2.1 is now complete except for the construction of the $U_{x}$ 's needed to decompose $\mathbf{Z}^{d}$ into the rooted directed trees, $T_{w}$, for $w \in S$.

Tree decomposition of $\mathbf{Z}^{d}$. We are given $\left\{c_{y}: y \in \mathbf{Z}^{d}\right\}$ satisfying Eq. (2.6) and a nonempty subset $S$ of $\mathbf{Z}^{d}$ satisfying Eq. (2.7). The object is to construct $U_{x}$ 's for $x$ in $\mathbf{Z}^{d}$, which partition $\mathbf{Z}^{d} \backslash S$ and hence construct the rooted directed trees $T_{w}$, for $w$ in $S$ which partition $\mathbf{Z}^{d}$ in such a way that Eqs. (3.1) and (3.4) are valid. Before doing this in general, let us consider a simpler context in which $\mathbf{Z}^{d}$ is replaced by the natural numbers $\mathbf{N}=\{1,2,3, \ldots\}$ and in which $c_{y}=|y|^{-\alpha}\left(c_{0}=0\right)$ with $1 / 2<\alpha \leqq 1$.

The construction in this simple context will serve both as an illustration and as a tool for the general context. We express $S$ as $\left\{w_{0}, w_{1}, w_{2}, \ldots\right\}$ with $w_{j}<w_{j+1}$ and note that in our simple context, Eq. (2.7) reduces to

$$
\sum_{j=0}^{\infty}\left(w_{j}\right)^{-\alpha}<\infty
$$

Our construction will be based on successive halving. We begin by defining successively halved subsets of $\mathbf{N}$ :

$$
\begin{aligned}
G_{0} & =\{1,3,5,7, \ldots\}=\{2 n-1: n \in \mathbf{N}\}, \\
G_{1} & =\{2,6,10, \ldots\}=\{4 n-2: n \in \mathbf{N}\} \\
& \vdots \\
G_{k} & =\left\{2^{k+1} n-2^{k}: n \in \mathbf{N}\right\} .
\end{aligned}
$$


For $k \geqq 1, T_{w_{k}} \backslash\left\{w_{k}\right\}$ will be a subset of $G_{k}$, but because of Eq. (3.4) we will want it to include only fairly large integers, i.e., ones at least as large as $L_{k}$, defined by

$$
\begin{aligned}
L_{k} & =\min \left\{n \in \mathbf{N}:\left[\sum_{y=n}^{\infty}|y-x|^{-2 \alpha}\right]^{1 / 2} \leqq k^{-2} \text { for all } x \leqq k\right\} \\
& =\min \left\{n \geqq k: \sum_{y=n}^{\infty}(y-k)^{-2 \alpha} \leqq k^{-4}\right\} .
\end{aligned}
$$

(We remark that $k^{-2}$ in the intermediate expression of Eq. (3.16) can be replaced by any summable sequence.) We define

$$
\begin{aligned}
F_{i} & =G_{i} \cap\left\{n: n \geqq L_{i}\right\} \text { for } i \geqq 1, \\
F_{0} & =\mathbf{N} \backslash\left(\bigcup_{i=1}^{\infty} F_{i}\right) ;
\end{aligned}
$$

for each $i \geqq 0, T_{w_{i}} \backslash\left\{w_{i}\right\}$ will be exactly $F_{i} \backslash S$. For each $i$, we must define $U_{x}$ for each $x$ in $T_{w_{i}}$. Put the integers of $F_{i} \backslash S$ in increasing order, $x_{1}^{(i)}<x_{2}^{(i)}<\ldots$, and set $x_{0}^{(i)}=w_{i}$. Then define the $U_{x}$ 's by successive halving:

$$
\begin{gathered}
U_{w_{i}}=U_{x_{0}^{(i)}}=\left\{x_{j}^{(i)}: j \in G_{0}\right\}=\left\{x_{1}^{(i)}, x_{3}^{(i)}, x_{5}^{(i)}, \ldots\right\} \\
U_{x_{1}^{(i)}}=\left\{x_{j}^{(i)}: j \in G_{1}\right\}=\left\{x_{2}^{(i)}, x_{6}^{(i)}, x_{10}^{(i)}, \ldots\right\} \\
\vdots \\
U_{x_{k}^{(i)}}=\left\{x_{j}^{(i)}: j \in G_{k}\right\} .
\end{gathered}
$$

In our simple context Eq. (3.1) reduces to $\sum_{y \in U_{x}} y^{-\alpha}=\infty$. To see that this is valid, first note that $\sum_{y \in G_{k}} y^{-\alpha}=\infty$ for each $k$ and hence $\sum_{y \in F_{2}} y^{-\alpha}=\infty$ (since $F_{0} \supseteq G_{0}$ and for $i \geqq 1, F_{i}$ and $G_{i}$ differ by only finitely many elements.) By Eq. (3.14), also $\sum_{y \in F_{\backslash} \backslash S} y^{-\alpha}=\infty$. Finally, by the monotonicity of $y^{-\alpha}$, the construction (3.18) implies that for $k \geqq 0$,

$$
-\left(x_{2^{k}}^{(i)}\right)^{-\alpha}+\sum_{y \in U_{x_{k}^{(i)}}} y^{-\alpha} \leqq \sum_{l=k+1}^{\infty} \sum_{y \in U_{x_{l}^{(i)}}} y^{-\alpha} \leqq \sum_{y \in U_{x_{k}^{(i)}}} y^{-\alpha},
$$

(where $x_{2^{k}}^{(i)}$ is the first element of $U_{x_{k}^{(i)}}$ ) which implies that

$$
\sum_{y \in U_{x_{k}^{(i)}}} y^{-\alpha}=\infty \quad \text { for each } k \geqq 0,
$$

as desired.

The condition (3.4) reduces to

$$
\sum_{k=0}^{\infty}\left[w_{k}^{-2 \alpha}+\sum_{\substack{y \in F_{k} \backslash S \\ y \neq x}}|y-x|^{-2 \alpha}\right]^{1 / 2}<\infty .
$$

Using $\sqrt{a^{2}+b^{2}} \leqq|a|+|b|$, we bound the LHS of Eq. (3.21) by

$$
\sum_{k=0}^{\infty} w_{k}^{-\alpha}+\sum_{k=0}^{L_{x}-1}\left[\sum_{y \neq x}|y-x|^{-2 \alpha}\right]^{1 / 2}+\sum_{k=L_{x}}^{\infty}\left[\sum_{y \in F_{k} \backslash S}|y-x|^{-2 \alpha}\right]^{1 / 2} \text {. }
$$


The first term is finite by Eq. (3.14), the second because $\alpha>1 / 2$, and the third is bounded (see Eq. (3.16)) by

$$
\sum_{k=L_{x}}^{\infty}\left[\sum_{y \geqq L_{x}}|y-x|^{-2 \alpha}\right]^{1 / 2} \leqq \sum_{k=L_{x}}^{\infty} k^{-2}<\infty .
$$

Thus Eq. (3.4) is verified in the simple context.

We proceed to the general context. The main complication is the absence of any monotonicity for the coefficients $\left\{c_{y}: y \in \mathbf{Z}^{d}\right\}$. Roughly speaking, we will circumvent this by grouping sites of $\mathbf{Z}^{d} \backslash S$ together in shells,

$$
\begin{aligned}
& B_{1}=\Lambda_{M_{1}} \backslash S, \\
& B_{k}=\left(\Lambda_{M_{k}} \backslash \Lambda_{M_{k-1}}\right) \backslash S \text { for } k \geqq 2,
\end{aligned}
$$

where the $M_{k}$ 's are defined sequentially as

$$
\begin{aligned}
M_{1} & =\min \left\{n: \sum_{y \in \Lambda_{n} \backslash s}\left|c_{y}\right| \geqq 1\right\}, \\
M_{k+1} & =\min \left\{n>M_{k}: \sum_{y \in\left(\Lambda_{n} \backslash \Lambda_{M_{k}}\right) \backslash S}\left|c_{y-x}\right| \geqq 1 \text {, for all } x \in \Lambda_{M_{k}}\right\} .
\end{aligned}
$$

Each $U_{x}$ will be a union of shells, $B_{k}$; the $B_{k}$ 's will be chosen much like the individual sites were chosen in our simpler context. $G_{i}$ and $F_{i}$ for $i \geqq 0$ are defined exactly as before, by Eqs. (3.15) and (3.17), except that the definition (3.16) for $L_{k}$ is replaced by

$$
\begin{aligned}
L_{k} & =\min \left\{n>k:\left[\sum_{y \notin \Lambda_{M_{n-1}}}\left|c_{y-x}\right|^{2}\right]^{1 / 2} \leqq k^{-2} \text { for all } x \in \Lambda_{M_{k}}\right\} \\
& =\min \left\{n>k: \max _{x \in \Lambda_{M_{k}}} \sum_{y \notin \Lambda_{M_{n-1}}}\left|c_{y-x}\right|^{2} \leqq k^{-4}\right\} .
\end{aligned}
$$

At this stage, we need some orderings. For each $i \geqq 0$, put the integers of $F_{i}$ in increasing order, $f_{1}^{(i)}<f_{2}^{(i)}<\ldots$ Choose any ordering for $S$ so that $S=\left\{w_{0}, w_{1}, w_{2}, \ldots\right\} ; T_{w_{i}} \backslash\left\{w_{i}\right\}$ will be exactly $\bigcup_{j \in F_{i}} B_{j}$. To construct the $U_{x}$ 's, we choose a "spiral" ordering of $\mathbf{Z}^{d}$ so that for every $n$, all sites $x \in \Lambda_{n}$ come before all sites $x \notin \Lambda_{n}$. We then use this fixed ordering on $\mathbf{Z}^{d}$ to order, for each $i$, the sites in

$$
\bigcup_{j \in F_{\imath}} B_{j}=\left\{x_{1}^{(i)}, x_{2}^{(i)}, x_{3}^{(i)}, \ldots\right\}
$$

and we set $x_{0}^{(i)}=w_{i}$. The construction (3.18) is now replaced by

$$
\begin{aligned}
U_{w_{i}} & =U_{x_{0}^{(i)}}=\bigcup_{j \in G_{0}} B_{f_{j}^{(i)}}=B_{f_{1}^{(i)}} \cup B_{f_{3}^{(i)}} \cup B_{f_{5}^{(i)}} \cup \ldots \\
& \vdots \\
U_{x_{k}^{(i)}} & =\bigcup_{j \in G_{k}} B_{f_{j}^{(i)}} .
\end{aligned}
$$

We have now defined all the $U_{x}$ 's and hence all the $T_{w}$ 's. To verify Eq. (3.1), observe that $U_{x}$ is a union of infinitely many $B_{k}$ 's. It follows from Eqs. (3.24)-(3.25) 
that for any given $x, \sum_{y \in B_{k}}\left|c_{y-x}\right| \geqq 1$ for all large $k$, and hence Eq. (3.1) is valid. The verification of Eq. (3.4) is essentially the same as the analogous verification of Eq. (3.21) done in the simpler context. The construction of our tree decomposition, and the proof of Theorem 2.1, is now completed.

\section{Proof of Theorem 2.2}

We begin by noting that to prove that the event defined at the end of the theorem has probability one, it of course suffices to show that for any $\varepsilon>0$, its probability is at least $1-\varepsilon$. Henceforth, letting $\varepsilon>0$ be fixed, we will construct our configuration $\tilde{\sigma}=\tilde{\sigma}(\hat{\sigma})$ in an $\varepsilon$-dependent way and will show that

$$
P(\text { for all } \hat{\sigma}, \tilde{\sigma}(\hat{\sigma}) \text { is a pliable, } \theta \text {-stiff ground state }) \geqq 1-\varepsilon \text {. }
$$

Next we relate $\theta$-stiffness (and pliability) of $\sigma$ to the values of $\Delta \mathscr{H}_{A}(\sigma)$ for $A$ a singleton set $\{x\}$, i.e., to the $\Delta \mathscr{H}_{x}(\sigma)$ 's defined in Eq. (2.4). For any finite $A$,

$$
\Delta \mathscr{H}_{A}(\sigma)=\sum_{x \in A} \Delta \mathscr{H}_{x}(\sigma)-\sum_{x, y \in A} J_{x y} \sigma_{x} \sigma_{y} \geqq \sum_{x \in A} \Delta \mathscr{H}_{x}(\sigma)-\sum_{x, y \in A}\left|J_{x y}\right| .
$$

From this we observe two things. First $\tilde{\sigma}$ will be pliable if each $\Delta \mathscr{H}_{x}(\tilde{\sigma})$ exists and is finite. Second, let us order the sites of $\mathbf{Z}^{d}$ (e.g., by the "spiral" ordering discussed near the end of Sect. 3) as $x_{1}, x_{2}, x_{3}, \ldots$ and define for $k \geqq 1$,

$$
\theta_{k}=\max \left\{\theta_{A}: x_{k} \in A \text { but } x_{j} \notin A \text { for } j>k\right\} ;
$$

then $\tilde{\sigma}$ is $\theta$-stiff if (recall that $\theta_{A} \geqq 0$ for each $A$ )

$$
\Delta \mathscr{H}_{x_{k}}(\tilde{\sigma})-2 \sum_{j=1}^{k-1}\left|J_{x_{j} x_{k}}\right| \geqq \theta_{k} \quad \text { for all } k \geqq 1 .
$$

Let $\phi_{k}, k \geqq 1$, be some fixed numbers such that $\phi_{k}>0, \sum \phi_{k}=1$ (e.g., $\phi_{k}=2^{-k}$ ). Then to obtain Eq. (4.1), it suffices to show that for all $k \geqq 1$,

$$
P\left(\text { for all } \hat{\sigma}, \infty>\Delta H_{x_{k}}(\tilde{\sigma}(\hat{\sigma})) \geqq 2 \sum_{j=1}^{k-1}\left|J_{x_{j} x_{k}}\right|+\theta_{k}\right) \geqq 1-\varepsilon \phi_{k} .
$$

It should be understood that the event in Eq. (4.5) includes the existence of the limit defining $\Delta \mathscr{H}_{x_{k}}$. We will obtain Eq. (4.5) by choosing constants $\lambda_{k}$ (with $\lambda_{1}=0$ ) and showing that

$$
\begin{gathered}
P\left(2 \sum_{j=1}^{k-1}\left|J_{x_{j} x_{k}}\right| \geqq \lambda_{k}\right) \leqq \varepsilon \phi_{k} / 2, \\
P\left(\text { for all } \hat{\sigma}, \infty>\Delta H_{x_{k}}(\tilde{\sigma}(\hat{\sigma})) \geqq \lambda_{k}+\theta_{k}\right) \geqq 1-\varepsilon \phi_{k} / 2 .
\end{gathered}
$$

By Chebyshev's inequality (and the Cauchy-Schwarz inequality which gives $\left.E\left(\left|Z_{x y}\right|\right) \leqq\left[E\left(\left|Z_{x y}\right|^{2}\right)\right]^{1 / 2}=1\right)$, the LHS of Eq. (4.6) is bounded by

$$
E\left(2 \sum_{j=1}^{k-1}\left|J_{x_{j} x_{k}}\right|\right) / \lambda_{k} \leqq 2\left(\sum_{j=1}^{k-1}\left|c_{x_{k}-x_{j}}\right| \cdot E\left(\left|Z_{x_{k} x_{j}}\right|\right)\right) / \lambda_{k} \leqq 2 \mathscr{C}_{k-1} / \lambda_{k}
$$


where $\mathscr{C}_{k}$ denotes the sum of the $k$ largest $\left|c_{j}\right|$ 's. Thus we obtain Eq. (4.6) by choosing, for $k \geqq 2$,

$$
\lambda_{k}=4 \mathscr{C}_{k-1} / \varepsilon \phi_{k} .
$$

It remains to construct $\tilde{\sigma}(\hat{\sigma})$ so that Eq. (4.7) is valid for each $k \geqq 1$ with our given and chosen $\lambda_{k}, \theta_{k}$, and $\phi_{k}$. To simplify the notation a bit, let us define $\rho_{x}$ and $\varepsilon_{x}$ for each $x$ in $\mathbf{Z}^{d}$ by setting $\rho_{x}=\lambda_{k}+\theta_{k}$ and $\varepsilon_{x}=\varepsilon \phi_{k} / 2$ with $k$ chosen so that $x_{k}=x$. Let us also write $\Delta \mathscr{H}_{x}(\hat{\sigma})$ for $\Delta \mathscr{H}_{x}(\tilde{\sigma}(\hat{\sigma}))$. Then Eq. (4.7) becomes

$$
P\left(\text { for all } \hat{\sigma}, \infty>\Delta H_{x}(\hat{\sigma}) \geqq \rho_{x}\right) \geqq 1-\varepsilon_{x} \text {. }
$$

We will show that, for any given $\rho_{x}$ 's and any positive $\varepsilon_{x}$ 's we can construct $\tilde{\sigma}(\hat{\sigma})$ so that Eq. (4.10) is valid for each $x$ in $\mathbf{Z}^{d}$.

We use the same general strategy as in the proof of Theorem 2.1. Namely we decompose $\mathbf{Z}^{d}$ as a disjoint union of directed rooted trees, $T_{w}$, one for each $w \in S$ with $w$ the root of $T_{w}$, and then define $\tilde{\sigma}_{y}=\hat{\sigma}_{w} \eta_{y}$ for each $w$ and each $y \in T_{w}$ (as in Eqs. (3.2)-(3.3)). In fact, the set of sites in each $T_{w}$ will be precisely the same as that used for the proof of Theorem 2.1. Namely

$$
T_{w_{i}}=\left\{w_{i}\right\} \cup\left(\bigcup_{j \in F_{i}} B_{j}\right)
$$

with $\left\{w_{0}, w_{1}, \ldots\right\}$ any ordering of $S$, with the shells $B_{k}$ defined by Eqs. (3.24)(3.25), and with the $F_{i}$ 's $\left(G_{i}\right.$ 's and $L_{i}$ 's) defined by (3.17) ((3.15) and (3.26)). The difference comes in the construction of the $U_{x}$ 's $\left(U_{x}\right.$ is the set of "children" of $x$ in our tree structure). Once again each $U_{x}$ will be a union of the shells $B_{k}$, but this time it will be a finite union (chosen below).

The proof of Theorem 2.1 shows (see especially (3.6)-(3.10))

$$
\Delta \mathscr{H}_{x}(\hat{\sigma})=V_{x}^{\prime}+\sum_{w \in S} \hat{\sigma}_{w(x)} \hat{\sigma}_{w} V_{x, w}^{(\infty)}
$$

where $w(x)$ is the root whose tree contains $x$,

$$
V_{x}^{\prime}=\sum_{y \in U_{x}}\left|J_{x y}\right|+\left\{\begin{array}{l}
0, \text { if } x \in S \\
\left.\left|J_{y_{0} x}\right| \text { (where } x \in U_{y_{0}}\right), \text { otherwise }
\end{array}\right\}
$$

and

$$
V_{x, w}^{(\infty)}=\lim _{n \rightarrow \infty} \sum_{y \in \tilde{T}_{w \cap} A_{n}} c_{x-y} Z_{x-y} \eta_{x} \eta_{y}
$$

where $\tilde{T}_{w}=T_{w}$ for $w \neq w(x)$ and $\tilde{T}_{w(x)}$ is $T_{w(x)} \backslash\left\{y\right.$ 's and $y_{0}$ of (4.13) $\}$. The existence and finiteness (with probability one) of $V_{x, w}^{(\infty)}$ and of the sum in Eq. (4.12) follow from the proof of Theorem 2.1 (see (3.11)-(3.13)) and since $U_{x}$ is now finite, we have $\Delta \mathscr{H}_{x}(\hat{\sigma})$ existing and finite with probability one.

For a lower bound, we have

$$
\Delta \mathscr{H}_{x}(\hat{\sigma}) \geqq \sum_{y \in U_{x}}\left|J_{x y}\right|-\sum_{w \in S}\left|V_{x, w}^{(\infty)}\right|
$$

Since the RHS does not depend on $\hat{\sigma}$, we will obtain Eq. (4.10) by choosing the $U_{x}$ 's so that the RHS is at least $\rho_{x}$ with probability at least $1-\varepsilon_{x}$. First, we note that (by 
the martingale convergence theorem used in the proof of Theorem 2.1), the limit in Eq. (4.14) is both a.s. and in $L^{2}$ so that (compare Eq. (3.13))

$$
E\left[\left|V_{x, w}^{(\infty)}\right|^{2}\right]=\lim _{n \rightarrow \infty} E\left[\left|V_{x, w}^{(n)}\right|^{2}\right]=\sum_{y \in \widetilde{T}_{w}}\left|c_{x-y}\right|^{2} \leqq \sum_{y \in T_{w}}\left|c_{x-y}\right|^{2} .
$$

Thus for any $\mu_{x}>0$,

$$
\begin{aligned}
P\left(\sum_{w \in S}\left|V_{x, w}^{(\infty)}\right| \geqq \mu_{x}\right) & \leqq\left(\mu_{x}\right)^{-1} \cdot E\left[\sum_{w \in S}\left|V_{x, w}^{(\infty)}\right|\right] \leqq\left(\mu_{x}\right)^{-1} \cdot \sum_{w \in S}\left[E\left|V_{x, w}^{(\infty)}\right|^{2}\right]^{1 / 2} \\
& \leqq\left(\mu_{x}\right)^{-1} \cdot \sum_{w \in S}\left[\sum_{y \in T_{w}}\left|c_{y-x}\right|^{2}\right]^{1 / 2}
\end{aligned}
$$

where the last expression is finite by Eq. (3.4). Let us choose

$$
\mu_{x}=\left(\frac{2}{\varepsilon_{x}}\right) \sum_{y \in S}\left[\sum_{y \in T_{w}}\left|c_{y-x}\right|^{2}\right]^{1 / 2}
$$

then by Eq. (4.15), we will obtain Eq. (4.10) if we choose the $U_{x}$ 's so that for every $x$ in $\mathbf{Z}^{d}$

$$
P\left(\sum_{y \in U_{x}}\left|J_{x y}\right| \leqq \rho_{x}+\mu_{x}\right) \leqq \varepsilon / 2 .
$$

Let $\tilde{V}_{x}$ denote the random variable appearing in Eq. (4.19). We use the inequalities and identities,

$$
\begin{aligned}
P\left(\tilde{V}_{x} \leqq E\left[\tilde{V}_{x} / 2\right]\right) & \geqq P\left(\left|\tilde{V}_{x}-E\left[\tilde{V}_{x}\right]\right| \geqq E\left[\tilde{V}_{x} / 2\right]\right) \leqq \operatorname{Var}\left(\tilde{V}_{x}\right) /\left(E\left[\tilde{V}_{x} / 2\right]\right)^{2}, \\
E\left[\tilde{V}_{x}\right] & =\left(\sum_{y \in U_{x}}\left|c_{x-y}\right|\right) E[|Z|],
\end{aligned}
$$

where $Z$ has the same distribution as the $Z_{x y}$ 's, and

$$
\begin{aligned}
\operatorname{Var}\left(\tilde{V}_{x}\right) & =\sum_{y \in U_{x}} \operatorname{Var}\left(\left|J_{x y}\right|\right)=\sum_{y \in U_{x}}\left|c_{x-y}\right|^{2} \operatorname{Var}(|Z|) \\
& \leqq \sum_{y \in \mathbf{Z}^{d}}\left|c_{x-y}\right|^{2} E\left[|Z|^{2}\right]=\sum_{y \in \mathbf{Z}^{d}}\left|c_{y}\right|^{2},
\end{aligned}
$$

to conclude that Eq. (4.19) will be valid if both of the following inequalities are valid:

$$
\begin{gathered}
\left(\frac{1}{2} \sum_{y \in U_{x}}\left|c_{y-x}\right|\right) \cdot E[|Z|] \geqq \rho_{x}+\mu_{x}, \\
\left(\sum_{y \in Z^{d}}\left|c_{y}\right|^{2}\right) /\left[\left(\frac{1}{2} \sum_{y \in U_{x}}\left|c_{y-x}\right|\right) \cdot E[|Z|]\right]^{2} \leqq \varepsilon_{x} / 2 .
\end{gathered}
$$

Thus we simply need to choose each $U_{x}$ so that

$$
\sum_{y \in U_{x}}\left|c_{y-x}\right| \geqq v_{x} \equiv \frac{2}{E[|Z|]} \max \left(\rho_{x}+\mu_{x},\left[\left(2 / \varepsilon_{x}\right) \sum_{y \in \mathbf{Z}^{d}}\left|c_{y}\right|^{2}\right]^{1 / 2}\right) .
$$

This condition is the replacement of Eq. (3.1).

As mentioned above, $U_{x}$ will be a finite union of the shells $B_{k}$, with indices from $F_{i}$ when $x \in T_{w_{i}}$ (see Eq. (4.11)). To choose the indices, put the integers of each $F_{i}$ in 
increasing order, $f_{1}^{(i)}<f_{2}^{(i)}<\ldots$ and then (as in Eq. (3.27)) use the "spiral" ordering on $\mathbf{Z}^{d}$ to order the sites in each

$$
T_{w_{i}}=\left\{x_{0}^{(i)}=w_{i}, x_{1}^{(i)}, x_{2}^{(i)}, \ldots\right\} .
$$

Recalling that (as explained following Eq. (3.28) above) for any $x, \sum_{y \in B_{k}}\left|c_{y-z}\right| \geqq 1$ for all large $k$, we see that for any $x, \sum_{y \in U_{x}}\left|c_{y-x}\right|$ can be made larger than any given $v_{x}$ simply by taking $U_{x}$ to be a union of sufficiently many $B_{k}$ 's. To see concretely how we can thus satisfy Eq. (4.25), define for each $i \geqq 0$ and $k \geqq 0$,

$$
U_{x_{k}^{(i)}}=\bigcup_{j=R_{k-1}^{(i)}+1}^{R_{k}^{(i)}} B_{f_{j}^{(i)}}
$$

where for each $i \geqq 0$, the $R_{k}^{(i)}$ 's are defined inductively by $R_{-1}^{(i)}=0$ and

$$
R_{k}^{(i)}=\min \left\{R: R>R_{k-1}^{(i)} \text { and } \sum_{j=R_{k-1}^{(i)}+1}^{R} \sum_{y \in B_{f_{j}^{(i)}}}\left|c_{y-x_{k}^{(i)}}\right| \geqq v_{x}\right\} \text {. }
$$

This completes the proof of Theorem 2.2.

\section{Proof of Theorem 2.4}

Once again the proof is based on a tree structure. This time there will be a single tree. Its root will be $x_{1}$, the first site in the "spiral" ordering of $\mathbf{Z}^{d}$, and again $U_{x}$ will denote the immediate children of $x$ on the tree. Given $\sigma$, $\sigma^{\prime}$ will be defined by the requirement that

$$
\text { for all } x, \sigma_{y}^{\prime}=\sigma_{x} \operatorname{sgn}\left(J_{x y}\right) \text { for all } y \in U_{x} \text {. }
$$

This defines $\sigma_{y}^{\prime}$ except on the root; there it can be taken as +1 . The two crucial properties we need of our tree are Eq. (3.1) and (as replacement for Eq. (3.4))

$$
\sum_{x^{\prime} \in \mathbf{Z}^{d}}\left[\sum_{y \in U_{x^{\prime}}}\left|c_{y-x}\right|^{2}\right]^{1 / 2}<\infty \quad \text { for all } x \in \mathbf{Z}^{d} .
$$

Let us show that these two properties imply that (with probability one) for every configuration $\sigma, \sigma^{\prime}(\sigma)$, defined by Eq. (5.1), forces $\sigma$. Equivalently, we must show that for each $x \in \mathbf{Z}^{d}$,

$$
P\left(\sigma_{x} h_{x}\left(\sigma^{\prime}(\sigma)\right)=+\infty \text { for all } \sigma\right)=1 .
$$

But

$$
\begin{aligned}
\sigma_{x} h_{x}\left(\sigma^{\prime}(\sigma)\right) & =\frac{1}{2} \sum_{y \in U_{x}}\left|J_{x y}\right|+\frac{1}{2} \sigma_{x} J_{x x_{1}}+\frac{1}{2} \sum_{x^{\prime} \neq x} \sigma_{x} \sigma_{x^{\prime}}\left[\sum_{y \in U_{x^{\prime}}} J_{x y} \operatorname{sgn}\left(J_{x^{\prime} y}\right)\right] \\
& \geqq \frac{1}{2} \sum_{y \in U_{x}}\left|J_{x y}\right|-\frac{1}{2}\left|J_{x x_{1}}\right|-\frac{1}{2} \sum_{x^{\prime} \neq x}\left|\sum_{y \in U_{x^{\prime}}} J_{x y} \operatorname{sgn}\left(J_{x^{\prime} y}\right)\right| \\
& =+\infty \text { (with probability one), }
\end{aligned}
$$

where the existence, finiteness or infiniteness of the various expressions follows from Eqs. (3.1) and (5.2) by essentially the same arguments which, in the proof of Theorem 2.1 , yielded $\Delta \mathscr{H}\left(\sigma^{*}\right)=+\infty$ for all $\hat{\sigma}$ (with probability one) as a consequence of Eqs. (3.1) and (3.4). It remains to construct our tree. 
Each $U_{x}$ will be a union of shells, $B_{k}^{\prime}$, which (because there is no $S$ to delete) are defined a little differently than the $B_{k}$ 's of Eq. (3.24). Namely,

$$
\begin{aligned}
& B_{1}^{\prime}=\Lambda_{M_{1}^{\prime}} \backslash\left\{x_{1}\right\}, \\
& B_{k}^{\prime}=\Lambda_{M_{k}^{\prime}} \backslash \Lambda_{M_{k-1}^{\prime}} \text { for } k \geqq 2,
\end{aligned}
$$

where

$$
\begin{aligned}
M_{1}^{\prime} & =\min \left\{n ; \sum_{y \in \Lambda_{n}}\left|c_{y-x_{1}}\right| \geqq 1\right\}, \\
M_{k+1}^{\prime} & =\min \left\{n>M_{k}^{\prime}: \sum_{y \in \Lambda_{n} \backslash \Lambda_{M_{k}^{\prime}}}\left|c_{y-x}\right| \geqq 1 \text { for all } x \in \Lambda_{M_{k}^{\prime}}\right\} .
\end{aligned}
$$

We then define $L_{k}$ by Eq. (3.26) except that $M_{n-1}$ and $M_{k}$ are replaced by $M_{n-1}^{\prime}$ and $M_{k}^{\prime}$. $G_{i}$ and $F_{i}$ are then defined by Eqs. (3.15) and (3.17). We now simply define for $i \geqq 0$ (recall that $\mathbf{Z}^{d}=\left\{x_{1}, x_{2}, x_{3}, \ldots\right\}$ ),

$$
U_{x_{i}}=\bigcup_{j \in F_{i-1}} B_{j}
$$

The verification that this construction satisfies Eqs. (3.1) and (5.2) is similar to the analogous argument in the proof of Theorem 2.1; we leave it as an exercise for any reader who is still hanging on.

\section{References}

1. Edwards, S., Anderson, P.W.: J. Phys. F 5, 965 (1975)

2. Binder, K., Young, A.P.: Rev. Mod. Phys. 58, 801 (1986) (Comprehensive review of theoretical and experimental work on spin glasses)

3. Kotliar, G., Anderson, P.W., Stein, D.L.: Phys. Rev. B 27, 602 (1983)

4. van Enter, A.C.D.: J. Phys. A 21, 1781 (1988)

5. Sherrington, D., Kirkpatrick, S.: Phys. Rev. Lett. 35, 1972 (1975)

6. Fisher, D.S., Huse, D.A.: Phys. Rev. Lett. 56, 1601 (1986)

7. Khanin, K.M., Sinai, Ya.G.: J. Stat. Phys. 20, 573 (1979)

8. Campanino, M., Olivieri, E., van Enter, A.C.D.: Commun. Math. Phys. 108, 241 (1987)

9. van Enter, A.C.D., Fröhlich, J.: Commun. Math. Phys. 98, 425 (1985)

10. Fröhlich, J., Zegarlinski, B.: Commun. Math. Phys. 110, 121 (1987)

11. Zegarlinski, B.: private communication

12. Newman, C.M., Stein, D.L.: Phys. Rev. B 46, 973 (1992)

13. Chung, K.L.: A Course in Probability Theory. 2. ed., NY: Academic Press 1974

14. Hall, P., Heyde, C.C.: Martingale Limit Theory and Its Application. New York: Academic Press 1980

Communicated by M. Aizenman 
